

\title{
Developing Purposeful AI Use Cases - A Structured Method and Its Application in Project Management
}

\author{
Peter Hofmann ${ }^{1}$, Jan Jöhnk ${ }^{1}$, Dominik Protschky ${ }^{2}$, and Nils Urbach ${ }^{3}$ \\ ${ }^{1}$ Project Group Business \& Information Systems Engineering of the Fraunhofer FIT, \\ University of Bayreuth, Bayreuth, Germany \\ \{peter.hofmann, jan.joehnk\} dfit.fraunhofer.de \\ ${ }^{2}$ University of Bayreuth, Bayreuth, Germany \\ dominik.protschkyeuni-bayreuth.de \\ ${ }^{3}$ FIM Research Center, University of Bayreuth, Bayreuth, Germany \\ nils.urbachefim-rc.de
}

\begin{abstract}
An appropriate problem-solution-fit is essential to develop purposeful artificial intelligence (AI) applications. However, in domains with an unintuitive problem-solution-fit, such as project management $(\mathrm{PM})$, organizations require methodological guidance. Hence, we propose a five-step method to develop organization-specific AI use cases: First, companies must consider the context factors technology, organization (in particular data and application domain), and environment. Second, companies must identify existing domain problems and AI solutions. Third, our method facilitates abstraction to understand the underlying nature of the identified problems and AI solutions. Fourth, our problem-solutionmatrix assists companies to match AI functions with the domain context. Fifth, companies derive necessary implications for the subsequent use case implementation. To construct and evaluate our method, we followed the design science research paradigm complemented by situational method engineering and based on 14 interviews. Our method addresses a relevant practical problem and contributes to identifying purposeful AI use cases in unintuitive application domains
\end{abstract}

Keywords: Artificial Intelligence, Project Management, Use Case Development, Design Science Research, Situational Method Engineering.

\section{$1 \quad$ Introduction}

Advances in computational resources, the high availability of data, and advances in machine learning, among others, amplify the commercialization of artificial intelligence (AI) applications in a wide range of business areas. Exemplary AI applications range from medical diagnosis [1] over virtual assistants [2] to autonomous vehicles [2]. AI's potential to foster competitive advantage drives organizations to put the identification of AI use cases at the core of their agendas. Thus, AI is no longer a primarily technical issue. For one thing, companies often lack a thorough understanding of AI $[3,4]$. For another thing, developing use cases is not just copying other organizations' use cases since AI use cases need to meet organization-specific context 
factors $[3,4]$. While some application areas offer an intuitive problem-solution-fit (e.g., predictive maintenance), other application areas come along with major managerial challenges.

An exemplary application area with an unintuitive problem-solution-fit is project management (PM) with its temporary effort to build a unique result [5]. Since learning from data is at the center of current $\mathrm{AI}$ applications [6,7], unique situations and the lack of project-specific data [8], contradicts the idea of using AI for PM. Rather, many successful decisions seem to relate to the project managers' knowledge [5]. However, the PM domain offers manifold opportunities for the application of AI which are mostly undiscovered in practice. Various approaches to solving the different PM problems exist [e.g., 8, 9], but previous work has only focused on specific applications in the field of AI to address particular problems of PM. Practitioners face the challenge to identify the right solution or to understand which solution suits their current problem best [8]. In other words, there exists a need to support companies in bridging the gap between their organizational problems and potential AI solutions. On a technology-independent level, both practice and literature have already discussed the identification of use cases. However, the existing technology selection and evaluation literature [10-12] does not consider AI's characteristics sufficiently. In addition, data's central role in AI applications requires special attention. To close this research gap, we pose the following research question:

\section{Which method chunk composition can support practitioners in developing purposeful AI use cases for the application in PM?}

To answer this research question, we develop a method to identify organizationspecific use cases for applying AI from both a problem and an opportunity perspective. Hence, the method engineering goal is to develop AI use cases considering the domain's context and AI's functions, i.e. purposeful use cases. We apply situational method engineering (SME) [13] within the design science research paradigm (DSR) [14] to develop our method.

\section{Theoretical Background}

\subsection{Artificial Intelligence in Project Management}

In order to describe the application of AI in PM, we follow the broad and established definition of Russell and Norvig, who describe AI as intelligent agents [6]. PM does not seem to be an intuitive application domain for AI because data is not as structured and obvious as in other domains. However, both research and existing products indicate the use of AI in PM. While these existing solutions address problems in PM, they are not sufficient for successful projects. In addition, a project manager needs knowledge and intuition about PM and the application domain [5]. Furthermore, the success of a project depends on the project manager's performance and personality [5].

Therefore, we screened existing literature and available PM software solutions to provide an exemplary overview of AI use cases in PM. So far, literature particularly covers the use of machine learning for prediction. However, there are also AI 
applications considering budget, prioritizing requirements, or risk assessment. For example, Tronto et al. [8] predict the effort of projects based on cost drivers and software size variables. For predicting, they use an artificial neuronal network and a linear regression approach. Vargas [15] aims at predicting the PM cost of a given project. Nayebi et al. [16] developed a decision support system using natural language processing to improve effort estimation, scoping, and assignment of change requests in software projects. For further research see, for example, [9, 17, 18].

Existing research is slowly having an impact on product offerings. Today, most AI applications in PM are chatbots [19]. Here, the use of AI is at the input and output interface, but not in the PM task itself. Consequently, the AI application, instead of humans or rule-based software, triggers the PM task. For example, the project assistant bot stratejos (https://stratejos.ai) supports project teams by sharing daily tasks, creating new tasks, or requesting people to finish missing issues. Another example is the redbooth bot (https://redbooth.com), which illustrates daily tasks and issues the team is working on. However, applications already exist that execute PM core tasks. PMOtto (https://pmotto.ai) is a virtual assistant that advises on a task's time, cost, and resources based on Machine Learning. Cloverleaf (https://cloverleaf.me) is an AI-based platform that supports gaining insights into how the project team members work. For instance, Cloverleaf indicates where to focus on skill development. Furthermore, Cloverleaf understands how to position people in project teams and therefore helps to assemble the best possible project team for a project.

In summary, literature and existing products already offer some value-adding opportunities for the use of AI in PM. But organizations often lack the understanding for the AI solutions' benefits and thus, for creating a solution-problem-fit. Hence, we see the need for a method to match organization-specific problems and AI solutions.

\subsection{Technology Evaluation and Selection}

Technology evaluation and selection is a critical task for organizations to stay competitive $[11,20]$. Technology selection is the process of identifying technologies and choosing the most appropriate of them [20, 21]. However, the rapid development of complex technology landscapes and the increasing complexity and dynamism in the business environment complicate the technology selection process [21, 22]. Existing technology selection approaches vary in their structure: There are filtering approaches $[11,12,22]$, hierarchical structure approaches combined with fuzzy concepts [10, 23], and step-by-step approaches $[20,21,24]$ to identify the appropriate technology.

Comprehensively, the existing technology selection approaches need a specific predefined application $[11,12]$. Identifying potential technologies and solutions is an important first step for many technology selection models [10, 12, 20, 23]. After the first step, technology selection literature often incorporates different context areas, such as technology, market, strategy, and customers [12, 20-22]. Both identifying technologies and analyzing context factors represent an important basis for technology selection. In contrast to the first two aspects, technology understanding is only mentioned explicitly in Fridgen et al. [24]. The major part of the considered methods incorporates criteria to evaluate the added value of the technology to be introduced [10, 
11,21-23]. Subsequently, existing technology selection literature suggests a structured comparison of the evaluated solutions following predetermined criteria [10, 12, 23].

Regarding successful technology implementation, AI's specifics require explicit consideration in organizations. First, organizations often do not fully understand AI's potential which may result in exaggerated expectations [4]. Consequently, it is important to develop an understanding of AI before identifying use cases. Second, data is indispensable for AI applications and is increasingly becoming a central resource for organizations. Thus, criteria of application, data, and organization must be aligned when using AI. If a use case does not comply with all of these criteria, AI's potential is at risk. Third, AI has a modular character $[25,26]$ that makes it very promising to transfer existing solutions to other domains and to recombine individual AI components to create completely new use cases. All three aspects are important for applying AI successfully but underrepresented in existing technology selection approaches.

Further, collecting and comparing the necessary information for technology selection is difficult [10]. Especially for new emerging technologies such as AI, comparable products or markets are scarce [27]. In contrast to existing technology selection approaches, companies increasingly face the challenge of finding reliable and appropriate use cases for emerging technologies. Fridgen et al. [24] describe an opportunity-oriented approach to develop blockchain use cases. Similar, AI has various application opportunities which is a why the problem perspective in technology selection is no longer sufficient. Thus, adding an opportunity perspective fosters the development of appropriate use cases, especially for domains with unintuitive problemsolution-fit.

Summarizing, the gap between existing technology selection approaches and AI's specifics is too large to apply them directly. Instead companies require an adapted approach taking AI's specifics and an opportunity perspective into consideration. Therefore, we use selected components of existing approaches to compose an appropriate method for developing purposeful AI use cases.

\section{Research Method}

We follow the DSR paradigm to develop our artifact, i.e., a method that identifies AI use cases in PM. DSR is an approach to develop and evaluate artifacts that serve human or organizational purposes rigorously [28, 29]. Methods, as an acknowledged DSR artifact, specify how to perform goal-directed activities [29]. We complement the DSR paradigm with principles of SME [13]. SME provides techniques to create IS development methods fulfilling the requirements of a given situation [30]. Previous scholars have shown the value of embedding SME within the DSR paradigm to rigorously develop methods [31, 32]. In our study, we use the assembly-based method engineering approach, a procedure model for SME proposed by Ralyté et al. [33]. The assembly-based method engineering approach allows us to make use of existing technology evaluation and selection techniques (i.e., method chunks). Further, the iterative manner of the assembly-based method engineering allows us to incorporate 
additional insights from expert interviews. Consequently, while drawing on existing technology selection methods, this research approach accounts for AI's specifics.

In the course of our research project, we conducted, recorded, transcribed, and analyzed 14 semi-structured interviews [34]. The first ten interviews provided overarching insights into the practical relevance and related challenges of applying AI in PM motivating us to create a method that develops AI use cases in a domain with an unintuitive problem-solution-fit. The insights of the remaining four interviews especially encompass our method's evaluation to ensure its applicability. Moreover, we revisited the first ten interviews during evaluation to verify that our method is in accordance with interviewees' expectations. We used expert sampling [35] to identify interviewees with AI and PM expertise from different industries and company sizes. Table 1 provides an overview of the experts' background.

Table 1. Overview of Expert Interviews

\begin{tabular}{|c|c|c|c|c|c|c|}
\hline$\#$ & Position & $\begin{array}{c}\text { Area of } \\
\text { Expertise }\end{array}$ & $\begin{array}{c}\text { Experience } \\
\text { in Years }\end{array}$ & Industry & $\begin{array}{c}\text { Company } \\
\text { Size } \\
\end{array}$ & $\begin{array}{c}\text { Interview } \\
\text { Duration } \\
\end{array}$ \\
\hline E1 & $\begin{array}{c}\text { Senior Data } \\
\text { Specialist }\end{array}$ & AI & $<5$ & Energy & $<50,000$ & 56 \\
\hline E2 & IT Project Manager & $\mathrm{AI}$ and $\mathrm{PM}$ & $5-10$ & IT Consulting & $<500$ & 74 \\
\hline E3 & Founder and CEO & AI & $<5$ & Software Start-up & $<50$ & 46 \\
\hline E4 & IT Solution Architect & AI & $5-10$ & ICT & $<100$ & 105 \\
\hline E5 & SVP and COO & $\mathrm{AI}$ and $\mathrm{PM}$ & $>10$ & Logistic & $>100,000$ & 89 \\
\hline E6 & $\begin{array}{l}\text { Head of Program } \\
\text { Management }\end{array}$ & PM & $5-10$ & Engineering & $<25,000$ & 63 \\
\hline E7 & Senior IT Consultant & PM & $<5$ & IT Consulting & $<500$ & 69 \\
\hline E8 & IT Program Manager & PM & $>10$ & $\begin{array}{l}\text { ICT and IT } \\
\text { Consulting }\end{array}$ & $>100,000$ & 78 \\
\hline E9 & IT Project Manager & PM & $>10$ & Automotive & $>100,000$ & 65 \\
\hline E10 & Senior Director & PM & $>10$ & Logistic & $>100,000$ & 86 \\
\hline E11 & $\begin{array}{l}\text { Head of Strategy \& } \\
\text { Innovation }\end{array}$ & $\mathrm{AI}$ and $\mathrm{PM}$ & $>10$ & $\begin{array}{c}\text { AI Research \& } \\
\text { Development }\end{array}$ & $<50$ & 59 \\
\hline E12 & Data Analyst & AI & $5-10$ & Software Start-up & $<50$ & 49 \\
\hline E13 & IT Project Manager & PM & $<5$ & Engineering & $<25.000$ & 72 \\
\hline E14 & IT Security Manager & $\mathrm{AI}$ and $\mathrm{PM}$ & $<5$ & Public Sector & $<100$ & 54 \\
\hline
\end{tabular}

In the first step of our method development, we defined the method engineering goals based on our assessment of AI's relevance and the shortcomings of existing technology selection models to address AI's specifics. In the second step, we identified method chunk requirements and overarching completion conditions. We set our completion conditions (CC) as follows: (CC1) The method assembly fulfills the targeted method engineering goals. (CC2) Each method chunk fulfills its requirements. (CC3) The method assembly considers a problem and potential perspective. In the third and fourth step, we selected and subsequently assembled method chunks until the method met all completion conditions. In the fifth step, we initially evaluated the method to demonstrate its inherent utility $[28,29]$. Thereby, we followed the evaluation criteria of March and Smith [29], i.e., ease-of-use, feasibility, effectiveness, efficiency, and generality. 


\section{A Method for Developing Purposeful Artificial Intelligence Use Cases in Project Management}

In this section, we describe our method to develop purposeful AI use cases in domains with an unintuitive problem-solution-fit. We use PM as an exemplary application domain in this research project. Our method consists of five steps, i.e., preparing, discovering, understanding, designing, and implementing. Thereby, our method considers expert opinions to cater to the practitioners' needs, such as understanding the technology, business value focus, use case evaluation, and implementation approaches, among others. Figure 1 summarizes the five method steps.

\begin{tabular}{|c|c|c|c|c|c|}
\hline & Preparing & Discovering & Understanding & Designing & Implementing \\
\hline تُ & $\begin{array}{c}\text { Collecting and } \\
\text { structuring } \\
\text { information about } \\
\text { three context } \\
\text { factors }\end{array}$ & $\begin{array}{l}\text { Collecting domain } \\
\text { problems and } \\
\text { existing AI } \\
\text { solutions }\end{array}$ & $\begin{array}{c}\text { Understanding } \\
\text { domain problems } \\
\text { and AI solutions } \\
\text { through } \\
\text { abstraction }\end{array}$ & $\begin{array}{l}\text { Matching domain } \\
\text { problems and } \\
\text { AI solutions in a } \\
\text { problem-solution- } \\
\text { matrix }\end{array}$ & $\begin{array}{l}\text { Deriving the } \\
\text { prerequisites for } \\
\text { the successful } \\
\text { implementation of } \\
\text { selected use cases }\end{array}$ \\
\hline 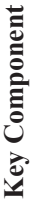 & $\begin{array}{l}\text { TOE framework } \\
\text { to consider the } \\
\text { context of } \\
\text { potential use cases }\end{array}$ & $\begin{array}{c}\text { Problem and } \\
\text { opportunity } \\
\text { perspective to } \\
\text { develop use cases } \\
\text { from two } \\
\text { points of view }\end{array}$ & $\begin{array}{c}\text { Domain structure, } \\
\text { AI functions and } \\
\text { solution types to } \\
\text { understand } \\
\text { AI characteristics } \\
\text { and its potential }\end{array}$ & $\begin{array}{l}\text { Problem-solution- } \\
\text { matrix to sort and } \\
\text { combine } \\
\text { identified } \\
\text { problems with } \\
\text { solutions }\end{array}$ & $\begin{array}{l}\text { Decision areas } \\
\text { and necessary } \\
\text { considerations for } \\
\text { purposful use case } \\
\text { implementation }\end{array}$ \\
\hline & $\begin{array}{l}\text { Requirements for } \\
\text { use cases from } \\
\text { technology, } \\
\text { organization, and } \\
\text { environment } \\
\text { context }\end{array}$ & $\begin{array}{l}\text { List of relevant } \\
\text { domain problems } \\
\text { and possible } \\
\text { AI solutions }\end{array}$ & $\begin{array}{c}\text { Abstract } \\
\text { assignment of } \\
\text { domain problems } \\
\text { and AI solutions } \\
\text { to problem and } \\
\text { solution classes }\end{array}$ & $\begin{array}{c}\text { Feasible list of use } \\
\text { cases with a } \\
\text { problem-solution- } \\
\text { fit }\end{array}$ & $\begin{array}{l}\text { Strategic and } \\
\text { contextual } \\
\text { implications for } \\
\text { use case } \\
\text { implementation }\end{array}$ \\
\hline
\end{tabular}

Figure 1. Method Overview for Developing AI Use Cases in the PM Application Domain

\subsection{Preparing}

The first method step (i.e., preparing) collects and structures relevant information about the organization-specific context. Thereby, we draw on technology selection literature $[10-12,20,22,23]$, the established Technology-Organization-Environment (TOE) framework [36], and AI adoption literature [3, 4] to derive relevant context factors for a detailed analysis. Technology selection is a critical process for organizations to stay competitive. By offering a generic theory for the diffusion of technologies, the TOE framework is suitable to get to know new technologies [37]. For example, Pumplun et al. [3] and AlSheibani et al. [4] describe AI adoption using the TOE framework and discuss context areas for using AI within an organization. To account for AI's and application domain's specifics we additionaly emphasize data and application domain as salient context areas within the TOE framework. Hence, we describe relevant aspects of the three context areas: technology, organization, and environment.

Technology. Organizations must provide an overall understanding of AI technologies. Further, organizations must consider the boundary conditions of existing 
strategies (business, IT, and AI, if applicable) for their technological implications as well as previous experience and current know-how within the organization regarding AI [3].

Organization. Considering the organization, aspects such as structures, resources, and culture play a decisive role [3]. It is important to ensure the alignment of emerging technologies such as AI with the organization's overarching strategy [21]. For instance, organizations must ensure, among others, top management support, an innovationfriendly culture, and adequate resources (e.g., budget, employee expertise, and availability).

Since the successful implementation of machine learning is especially reliant on data, we consider data as an integral resource [3]. For instance, training machine learning algorithms require large amounts of data to achieve adequate output quality [38]. However, data availability, quality, and security are often limiting factors in AI projects [3]. Besides, machine learning models can only generate meaningful output if data matches the application [6]. Therefore, organizations must carefully consider these aspects and evaluate the available data (e.g., actuality, relevance, accessibility).

With the method aiming at developing AI use cases within a specific application domain, organizations must assess the general domain characteristics. Thereby, organizations must gather relevant information and stakeholders of the application domain to create a shared understanding (e.g., through joint teams with AI and domain experts). For AI use cases in PM, this may include the total number of projects, information on project types, content, and size, success, and failures report or current tool support.

Environment. The environmental context includes an assessment of industry requirements, competitors, customers, and regulation [3]. Thereby, organizations must identify facilitating and impeding factors for AI use cases development. These factors may include general industry trends for AI adoption, customer preferences for AI acceptance, and the regulatory impact on AI implementation (e.g., public funding, GDPR and ethical guidelines on AI's trustworthiness [39]).

\subsection{Discovering}

The goal of the second method step (i.e., discovering) is to collect specific application domain problems and existing AI solutions. Thus, organizations can develop AI use cases from two perspectives: First, organizations may use AI to address existing problems in the application domain (problem perspective). Second, organizations may explore new AI solutions to open up new technological opportunities in the application domain (opportunity perspective).

Problem Perspective. In the PM case, following the problem perspective is a sensible approach to learn from past project failures for future projects [40]. However, the definitions of project success and failure differ in organizations as well as in literature [41]. Common success dimensions include the efficiency of the implementation process, the project's perceived value, and customer satisfaction [42]. Based on a common understanding of project failure, project managers must analyze their projects to identify past problems and impediments. This approach will initially 
result in very specific problems [40] which organizations can collate with PM failure literature [e.g., 40, 43-45].

Opportunity Perspective. The opportunity perspective identifies existing approaches and technological opportunities for AI use cases in the application domain. Companies must engage in a comprehensive market analysis [21] to collect existing AI solutions. Such analysis may include available products, potential technological opportunities, and research insights. Owing to the limited market of AI solutions, available products serve more as an inspiration for the development of use cases. Following this problem-agnostic approach, organizations gain a deeper understanding of potential AI solutions and can discover technological opportunities for additional improvements in PM [24].

\subsection{Understanding}

Organizations often lack profound knowledge of emerging technologies, which leads to exaggerated expectations [24]. Therefore, the third method step (i.e., understanding) further abstracts the identified domain problems and existing AI solutions to reveal their underlying nature. This understanding enables organizations to match problems and solutions in the subsequent method step. Moreover, it is important to understand the problems and solutions well to evaluate the application's outcome in the later development and deployment phase appropriately. Abstraction usually only changes the representation of a phenomenon (e.g., budget overrun in a specific project indicates a budget estimation problem) to reduce complexity [46]. Solving this abstraction is often easier than solving a specific phenomenon directly. Finally, one transfers the abstract solution into a specific solution for the original phenomenon (e.g., applying an AI-based budget estimation algorithm in a specific project with its characteristics). While an abstract solution can apply to several problems (e.g., several different projects with budget overruns), it is important to abstract purposefully to maintain the underlying nature of the phenomenon [47].

Application Domain Structure. Considering the domain understanding, organizations must find a way to structure the domain processes, activities, and expertise. For the PM application domain, we use the ten knowledge areas of the Project Management Body of Knowledge [5] as an expertise-focused de-facto PM standard. Organizations could also use process groups or PM phases to structure the application domain. However, we prefer the expertise-focus because it does not limit AI solutions to a specific project phase such as budget estimation at the beginning of a project or the ongoing prediction of budget needs. Of course, other application domains or organizations' specifics may require their own application domain structure (e.g., product development phases for AI uses in a production context).

AI Functions. Considering the AI understanding, we propose seven AI functions inspired by the human cognitive abilities for three major reasons. First, AI mimics problems of human thinking [6]. Second, AI is in part (e.g., artificial neural networks) inspired on the biological learning of humans [38]. Third, cognitive abilities are familiar to human decision-makers, facilitating the application of our method. For a discussion of cognitive abilities in PM see, among others, Mair et al. [48]. Consequently, we draw 
on psychology literature to distinguish the abstract tasks AI can support [e.g., 49, 50]. We list the seven AI functions, including a short definition, below.

- Perceiving, i.e., acquiring and processing data from the real world to produce information

- Feature extraction and identification, i.e., extracting and identifying specific objects from data

- Reasoning, i.e., explaining underlying relationships and structures in data

- Predicting, i.e., estimating future events or conditions on a continuous scale

- Decision-making, i.e., choosing between known, discrete alternatives

- Generating, i.e., producing or creating something

- Acting, i.e., executing goal-oriented actions (e.g., movement, navigate, control)

AI Solution Types. In addition to the AI functions, different AI solution types exist. We refined the solution type approaches of Auth et al. [19] and Lahmann et al. [51] and distinguish four AI solution types depending on the role, extent, and value of AI usage: rule-based solutions, AI-enabled solutions, AI-based solutions, and full AI solutions. Rule-based solutions do not possess any AI component but rely on common rule-based programming. Rule-based solutions are particularly useful for automating standardized project tasks via simple workflow integration (e.g., robotic process automation). AIenabled solutions use AI to support input and output interfaces. AI-enabled solutions usually comprise human-computer-interaction, often based on natural language processing (e.g., chatbots). AI-based solutions use AI to support processing the core task. Such solutions create new knowledge (e.g., budget estimation or risk management advice). Full AI solutions use AI for input and output as well as task processing. Full AI solutions may also consist of separate AI solutions (e.g., a chatbot that communicates AI-based budget estimations). Our method is primarily concerned with AI-based and full AI solutions because rule-based solutions to not include an AI component and AI-enabled solutions facilitate the process instead of addressing the core task. However, organizations can use this classification to guide the development of use cases for all four AI solution types.

\subsection{Designing}

The method's previous step resulted in a list of AI solutions and application domain problems as well as a deeper understanding of the application domain structure and AI functions. In the fourth method step (i.e., designing), one needs to match application domain problems and AI solutions by consolidating the gathered information in the problem-solution-matrix (cf. Figure 2): In the PM case, we organize the knowledge areas in the matrix's columns and the AI functions in the matrix's rows. Depending on which human ability would be necessary to solve the problem or which human ability resembles the solution, an assignment to the AI functions can take place. 


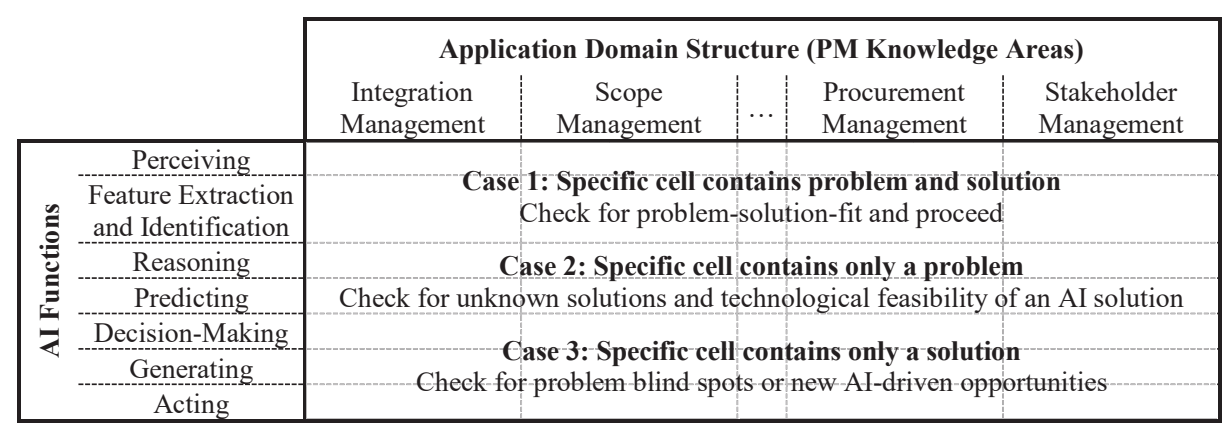

Figure 2. Problem-Solution-Matrix in the PM Application Domain

Problem-Solution-Constellations. After sorting the solutions and problems, three scenarios are possible: In the case of a problem-solution-fit, matrix elements include both problem(s) and solution(s). The organizations can compare the resulting use cases with the context factors and evaluate their added value. If there is only a problem in a matrix field, but no solution, the organization can conduct more intensive market research. The organization can search specifically for solutions in the area in which the problem occurred. If no solution exists, the organization can also evaluate whether developing the solution is possible. If there is only a solution in the matrix field, but no problem, the organization should check the following two possibilities. First, whether the organization has overlooked a problem. Second, if the organization has not overlooked a problem, whether the solution has the potential to improve current processes.

Comparison and Prioritization. As soon as a collection of use cases exists, the organization analyzes the context factors from the first method step (i.e., preparing) again, specifically for each use case. In this way, the organization can determine whether an implementation is possible at the current point in time or which obstacles and requirements exist to enable implementation. Based on this, the organization can prioritize the use cases. For this purpose, the organization must define criteria according to which it would like to evaluate the use cases. Common criteria from the literature refer to quality, cost, benefits, reliability, and compatibility [10, 22].

\subsection{Implementing}

The goal of the fifth method step (i.e., implementing) is to successfully take the hurdle from use case conception to use case implementation. Thus, organizations must put the theoretical considerations into practice and derive the prerequisites for successful use case implementation. Thereby, an organization must answer the question whether it wants to develop the AI application in-house, outsource the development, or purchase an existing solution from an external provider. Furthermore, organizations must consider the three context factors described in the first method step again during use case implementation. Regarding the technology context, the organization needs to consider, for example, how to integrate the AI application into the current infrastructure and how to develop a proof-of-concept. Closely related, the organization needs to 
consider the data context. For example, if the required data is not yet available, the organization must plan data acquisition or adapt its data strategy. In the organizational context, the organization needs to address, amongst others, employees' concerns. Regarding the domain context, the organization needs to decide how to change existing processes. As an example of the environmental context, the organization should verify data protection and ethical issues carefully.

\section{$5 \quad$ Evaluation and Discussion}

\subsection{Discussing the Method's Application}

As shown in section 2.2, the gap between existing technology selection approaches and AI's specifics requires an adaptation of existing approaches. The primary goal of technology selection is to compare different technology candidates and choose the most appropriate among them. In contrast, AI requires an alternative approach that combines a problem and opportunity perspective.

Our method is not an isolated approach, but organizations must explicitly consider its integration within the organizational context. AI is not just a topic for labs or the IT department. The resulting interdisciplinary teams naturally bring together a problem and opportunity perspective and thus, complement each other. Since AI use cases can affect many employees, change management becomes an important issue for organizations. To cope with that, our method allows incorporating non-AI-experts at the very beginning of developing AI use cases to shape the use of AI in their domain.

Moreover, our method may appear to follow a sequential order of method steps. However, we suggest a more iterative implementation. Since our method leaves room for tailored execution, one can apply our method to different extents. For example, AI experts, domain experts, and executives start with a workshop to generate a first rudimentary and quick assessment of use case areas. Insights from a first iteration allow planning further iterations in more detail. Furthermore, practitioners can apply our method in other domains with unintuitive problem-solution-fit. First, the introduced AI functions are applicable domain-independently because they do not have any specific domain characteristics. Second, the structure of our method allows replacing PM by other domains characterized by an unintuitive solution-problem-fit.

\subsection{Evaluating the Method's Utility}

Following March and Smith [29], we evaluate our method with regard to five criteria (i.e., ease-of-use, feasibility, effectiveness, efficiency, and generality) and discuss the fulfillment of the CC. Although we have not yet applied our method in a real-world scenario, we assess the method's utility initially based on the later expert interviews.

Feasibility. Overall, experts confirm our method's comprehensibility and feasibility [E11-E14]. Moreover, experts agree that all method steps are complete. Despite the uniqueness of projects, E11 states no further obstacles to use AI solutions across different projects. E12, E13, and E14 emphasize the applicability of our matrix. In 
detail, E12 can combine our AI functions with different AI algorithms and E13, as a non-AI-specialist, can comprehend the AI functions.

Ease-of-Use. Practitioners do not need to make any long-term investments to use our method. But our method implies that the organization must deal intensively with the domain and AI. However, from our point of view, this is necessary to develop purposeful use cases. Due to the method's variability, the execution effort is controllable and scalable. According to the organization's demands, the process can range from a one-day workshop to a longer phase with few participants or a whole team.

Efficiency. The method's iterative character gives practitioners the possibility to terminate the current method stage to avoid sunk cost. Iteratively executing our method allows one to get deeper insights into their problems to get solutions as purposeful as possible. E11 confirms that it is important to get solutions for specific problems in a purposeful way. Furthermore, the abstraction part of the method allows covering a certain number of problems with one solution.

Effectiveness. Overall, experts confirm the relevance of a structured approach to use AI solutions [E11-E14]. E12 points out the method's relevance by describing his thought to create a similar method for his organization to create solutions in order to be able to develop solutions rapidly and purposefully. For example, scope estimations [E13] are an application area for AI in PM, which our method can identify. However, E13 states that a high abstraction bears the risk of project managers sorting problems differently in the matrix.

Generality. As stated in section 5.1, the method is independent of the domain and thus, provides general applicability in organizations. While we introduce our method in a PM context, for example, E12 transfers the method - especially the AI functions to the medical sector. Furthermore, E14 transfers the method to a current IT security task and confirms the applicability of our matrix in this area.

Completion Conditions. Using our method, practitioners understand how to create AI use cases in PM. They can also organize AI knowledge and corresponding PM expertise ( $\mathrm{CC} 1)$. Furthermore, the experts confirm that each method chunk fulfills its requirements $(\mathrm{CC} 2)$. Lastly, the method considers a problem and a potential view, since sorting is possible from both perspectives (CC3). Consequently, the initial evaluation of our method indicates that our method meets the CC.

\subsection{Limitations and Future Research}

Our study is subject to limitations that stimulate future research. First, we use 14 expert interviews to support the entire method development process and its subsequent evaluation. While the interviewees' statements support the method's relevance, future research should extend the number of interview participants to extend our method's evaluation. Second, we cannot present a real-world scenario to evaluate our method. However, building on our insights during method development and its initial evaluation, an application of our method seems promising. Hence, future research may address detailed questions like team composition and reasonable method iterations when executing our method [E11]. Third, the rate of change and technological advancements in AI require continuous evaluation and improvement of our method. 
Fourth, future research may validate the application of our method in other domains with an unintuitive problem-solution-fit.

\section{Conclusion}

Motivated by organizations' need to understand the nature of AI and its value-adding application, we provide a five-step method to develop purposeful AI use cases. In particular, such a method is necessary for application areas that have an unintuitive problem-solution-fit. In this study, we used PM as an exemplary application area in which it seems especially difficult to assess AI's application potential. Our paper's theoretical contribution extends existing research on technology selection and use case identification by AI-specific methodological guidance. Moreover, we provide arguments for the generalizability of our method beyond PM to cover other application domains with an unintuitive problem-solution-fit. Further, our method holds important managerial implications by providing initial scientific guidance for a structured and thoughtful identification of AI use cases. Therefore, our method helps to demystify AI, its domain-specific application, and purposeful use cases.

\section{References}

1. Hofmann, P., Oesterle, S., Rust, P., Urbach, N.: Machine Learning Approaches along the Radiology Value Chain - Rethinking Value Propositions. Proceedings of the 27th European Conference on Information Systems (ECIS) (2019)

2. Rzepka, C., Berger, B.: User Interaction with AI-enabled Systems: A Systematic Review of IS Research. Proceedings of the 39th International Conference on Information Systems (ICIS) (2018)

3. Pumplun, L., Tauchert, C., Heidt, M.: A New Organizational Chassis for Artificial Intelligence - Exploring Organizational Readiness Factors. Proceedings of the 27th European Conference on Information Systems (ECIS) (2019)

4. AlSheibani, S., Cheung, Y., Messom, C.: Artificial Intelligence Adoption: AI-readiness at Firm-Level. Proceedings of the Twenty-Second Pacific Asia Conference on Information Systems (PACIS) (2018)

5. Project Management Institute: Guide to the project management body of knowledge. The Stationery Office Ltd, London (2018)

6. Russell, S., Norvig, P.: Artificial Intelligence. A Modern Approach. Pearson Education UK, Edinburgh (2016)

7. Bengio, Y., Courville, A., Vincent, P.: Representation learning: a review and new perspectives. IEEE transactions on pattern analysis and machine intelligence 35, 1798-1828 (2013)

8. Barcelos Tronto, I.F. de, da Silva, J.D.S., Sant'Anna, N.: An investigation of artificial neural networks based prediction systems in software project management. Journal of Systems and Software 81, 356-367 (2008)

9. Perini, A., Susi, A., Avesani, P.: A Machine Learning Approach to Software Requirements Prioritization. IEEE Transactions on Software Engineering 39, 445-461 (2013)

10. Chan, F.T.S., Chan, M.H., Tang, N.K.H.: Evaluation methodologies for technology selection. Journal of Materials Processing Technology 107, 330-337 (2000) 
11. Collins, M., Williams, L.: A Three-Stage Filter for Effective Technology Selection. Research-Technology Management 57:3, 36-42 (2014)

12. Yap, C.M., Souder, W.E.: A filter system for technology evaluation and selection. Technovation 13, 449-469 (1993)

13. Brinkkemper, S.: Method engineering: engineering of information systems development methods and tools. Information and Software Technology 38, 275-280 (1996)

14. Gregor, S., Hevner, A.R.: Positioning and presenting design science research for maximum impact. MIS Quarterly 37, 337-355 (2013)

15. Vargas, R.: Applying Neural Networks and Analogous Estimating to Determine the Project Budget. PMI Global Congress (2015)

16. Nayebi, M., Kabeer, S.J., Ruhe, G., Carlson, C., Chew, F.: Hybrid Labels are the New Measure! IEEE Software 35, 54-57 (2018)

17. Fauser, J., Schmidthuysen, M., Scheffold, B.: The Prediction of Success in Project Management. Predictive Project Analytics. Projektmanagement aktuell 26, 66-74 (2015)

18. Shivaji, S., Whitehead, E.J., Akella, R., Kim, S.: Reducing Features to Improve Code Change-Based Bug Prediction. IEEE Transactions on Software Engineering 39, 552-569 (2013)

19. Auth, G., Jokisch, O., Dürk, C.: Revisiting automated project management in the digital age - a survey of AI approaches. Online Journal of Applied Knowledge Management 7, 27-39 (2019)

20. Stillman, H.M.: How ABB Decides on the Right Technology Investments. ResearchTechnology Management 40, 14-22 (1997)

21. Friedrich, T., Overhage, S., Schlauderer, S., Eggs, H.: Selecting Technologies for Social Commerce: Towards a Systematic Method. Proceedings of the 23rd European Conference on Information Systems (ECIS) (2015)

22. Shehabuddeen, N., Probert, D., Phaal, R.: From theory to practice: challenges in operationalising a technology selection framework. Technovation 26, 324-335 (2006)

23. Shen, Y.-C., Chang, S.-H., Lin, G.T.R., Yu, H.-C.: A hybrid selection model for emerging technology. Technological Forecasting and Social Change 77, 151-166 (2010)

24. Fridgen, G., Lock1, J., Radszuwill, S., Rieger, A., Schweizer, A., Urbach, N.: A Solution in Search of a Problem - A Method for the Development of Blockchain Use. 24th Americas Conference on Information Systems (AMCIS) (2018)

25. Bitkom: Digitalisierung gestalten mit dem Periodensystem der Künstlichen Intelligenz. Ein Navigationssystem für Entscheider (2018)

26. Hammond, K.J.: The Periodic Table of AI, https://www.datasciencecentral.com/profiles/ blogs/the-periodic-table-of-ai (2017)

27. Christensen, C.M.: The Innovator's Dilemma. When New Technologies Cause Great Firms to Fail. Harvard Business Review Press, Boston (2013)

28. Hevner, A.R., March, S.T., Park, J., Ram, S.: Design Science in Information Systems Research. MIS Quarterly 28, 75-105 (2004)

29. March, S.T., Smith, G.F.: Design and natural science research on information technology. Decision Support Systems 15, 251-266 (1995)

30. Henderson-Sellers, B., Ralyté, J.: Situational Method Engineering: State-of-the-Art Review. Journal of Universal Computer Science 16, 424-478 (2009)

31. Denner, M.-S., Püschel, L.C., Röglinger, M.: How to Exploit the Digitalization Potential of Business Processes. Business \& Information Systems Engineering 60, 331-349 (2018)

32. Hofmann, P., Keller, R., Urbach, N.: Inter-technology relationship networks: Arranging technologies through text mining. Technological Forecasting and Social Change 143, 202$213(2019)$ 
33. Ralyté, J., Deneckère, R., Rolland, C.: Towards a Generic Model for Situational Method Engineering. In: Eder, J., Missikoff, M. (eds.) Advanced Information Systems Engineering. 15th International Conference, CAiSE 2003, 141, pp. 95-110. Springer, Berlin and Heidelberg, Germany (2003)

34. Myers, M.D., Newman, M.: The qualitative interview in IS research: Examining the craft. Information and Organization 17, 2-26 (2007)

35. Bhattacherjee, A.: Social Science Research: Principles, Methods, and Practices. Scholar Commons, University of South Florida, Tampa, Florida (2012)

36. Baker, J.: The Technology-Organization-Environment Framework. In: Dwivedi, Y.K., Wade, M.R., Schneberger, S.L. (eds.) Information Systems Theory, 28, pp. 231-245. Springer New York, New York, NY (2012)

37. Zhu, K., Kraemer, K.L.: Post-Adoption Variations in Usage and Value of E-Business by Organizations: Cross-Country Evidence from the Retail Industry. Information Systems Research 16, 61-84 (2005)

38. Neftci, E.O., Averbeck, B.B.: Reinforcement learning in artificial and biological systems. Nature Machine Intelligence 1, 133-143 (2019)

39. High-Level Expert Group on Artificial Intelligence: Ethics Guidelines for Trustworthy AI (2019)

40. Nelson, R.R.: IT Project Management - Infamous Failures Classic Mistakes, and Best Practices. MIS Quarterly Executive 6, 67-78 (2007)

41. Basten, D., Pankratz, O., Joosten, D.: Assessing the Assessors - An Overview And Evaluation Of IT Project Success Reports. Proceedings of the 21st European Conference on Information Systems (ECIS) (2013)

42. Pankratz, O., Basten, D.: Ladder to success - eliciting project managers' perceptions of IS project success criteria. International Journal of Informations Systems and Project Management 2, 5-24 (2014)

43. Belassi, W., Tukel, O.I.: A new framework for determining critical success/failure factors in projects. International Journal of Project Management 14, 141-151 (1996)

44. Pinto, J.K., Mantel, S.J.: The causes of project failure. IEEE Transactions on Engineering Management 37, 269-276 (1990)

45. Yeo, K.T.: Critical failure factors in information system projects. International Journal of Project Management 20, 241-246 (2002)

46. Zucker, J.-D.: A grounded theory of abstraction in artificial intelligence. Philosophical transactions of the Royal Society of London. Series B, Biological sciences 358, 1293-1309 (2003)

47. Saitta, L., Zucker, J.-D.: Abstraction in Artificial Intelligence and Complex Systems. Springer New York, New York, NY (2013)

48. Mair, C., Martincova, M., Shepperd, M.: An Empirical Study of Software Project Managers Using a Case-Based Reasoner. Proceedings of the 45th Hawaii International Conference on System Sciences (HICSS), 1030-1039 (2012)

49. Carroll, J.B.: Human cognitive abilities. A survey of factor-analytic studies. Cambridge Univ. Press, Cambridge (2004)

50. Gerrig, R., Zimbardo, P., Campbell, A., Cumming, S., Wilkes, F.: Psychology and Life. Pearson Education Australia, Melbourne (2012)

51. Lahmann, M., Keiser, P., Stierli, A.: AI will transform project management. Are you ready? (2018) 